\title{
A Method of Modeling of Security and Stability Control System Based on Virtual Relays
}

\author{
Guoyang $\mathrm{Wu}^{1, \mathrm{a}}$, Liang Yuan ${ }^{2, \mathrm{~b}}$, Xinli Song ${ }^{1, \mathrm{c}}$, Yanjia Liu ${ }^{1, \mathrm{~d}}$ \\ ${ }^{1}$ No.15 Xiaoying East Rd,Haidian,Beijing,China \\ ${ }^{2}$ No.619 Yonghua Notrh St,Baoding,China \\ awugy@epri.sgcc.com.cn, byliang@126.com, 'songxl@epri.sgcc.com.cn, diuyj@epri.sgcc.com.cn
}

Keywords: Security and stability control system, Modeling, Simulation, Virtual relay

\begin{abstract}
Security and stability control systems (SSCSs) are effective measures to ensure the safe operation of power grids. A normal SSCS has a complex structure and is specific targeted. Therefore, it is difficult to model SSCSs accurately in large scale power system dynamic simulation. A new modeling method of SSCS is put forward in this paper, which can accurately represent the dynamic performance of an actual SSCS. The method reduces a great deal of computation and is easy to use. It can be applied to simulate serious accidents and research anti-accident measures to blackouts caused by cascading failures. It can be an effective tool not only for studying dynamic characteristic and mechanisms of non-linear large scale power systems, but also for the accurate analysis of power system dynamic stability.
\end{abstract}

\section{Introduction}

Security and stability control systems (SSCSs) are effective measures to ensure the safe operation of power grids, and they are important defense lines to prevent system crash in cascading failures. The validity and accuracy of power system dynamic simulations would be improved by modeling security and stability control systems according to their operation characteristics.

SSCSs have complex structures with lots of start-up and latch-up and block circuits, and they are specifically targeted and different from each other. Although many scholars have studied on the modeling of SSCSs, most of them are limited to DTS [1-7]. Due to constraints of real-time, these models are typically simple, and the simulation results are not accurate enough compared with actual situation.

Although, the widely used simulation programs for transient stability of power system, such as PSS/E, PSASP and PSD-BPA, include some simple models such as under-frequency load shedding and under-voltage load shedding, there is no accurate and systematical modeling method for SSCSs, let alone the models based on foreign devices, which are greatly different from those used in China [8]. There are some cases of relay action which may provoked by transmission line overload, especially during blackouts caused by cascading failures. Therefore, these programs cannot satisfy the simulation requirement of the full dynamic process of power systems.

This paper puts forward a general modeling method for SSCSs based on virtual relays. Adopting an object-oriented strategy, the method combined with appropriate hierarchy simplification greatly reduces the complexity of modeling.

\section{Virtual relay [9]}

Though a real SSCS is very complex, it can be considered as a special relay system. Usually a relay device samples input signals and obtains the logical output signals " 0 " or " 1 " by comparing them with corresponding settings. Then, with appropriate logical decisions, protective devices indicate whether to operate or not. To describe the logical decision procedures clearly, we may consider every logical judge step as a simple relay, and call it a "virtual relay" because it does not actually exist. 
A current relay is a simple example which meets all the requirements of a virtual relay operating at a specified electrical quantity. If the input current exceeds its action setting, the current relay acts immediately to close its contacts. And it resets with its contacts open again only if the input current goes down below the reset setting. We call the setting the "reference", which takes the form of a setting or constant. All the relays share the same principle as the current relay except for different input signals.

For convenience, the input relay, time relay, impulse relay, auxiliary relay and output relay are defined. The input relay herein can be used to describe the relay operation characteristics, which are based on the input excitation such as current, voltage, etc. Other type of relays are normal logical relays. In addition, deferent types of out relay have to be defined for generating unit tripping, load shedding, fast valving, line cutting, capacitor and reactor switching, and so on.

\section{Modeling of SSCSs}

An SSCS is typically designed in a three-layer hierarchical structure. The first control layer contains one or more master stations, the second control layer consists of several sub-stations, and the third control layer is made up of a number of execution stations. Additional control layers can be used according to practical requirements.

Principles of modeling. An SSCS model is supposed to be made up of one master station and several sub-stations. The master station is responsible for the system management, such as recognizing the operation mode, collecting and exchanging information between stations, prioritizing load shedding and quantizing control measures. While sub-stations are responsible for message collection, fault detection, local or remote control. Besides, the execution stations no longer appear in a separate layer with their function integrated in the higher level sub-station. Thus, the simplified model of SSCS has a clear logical relationship and is easy to achieve.

Modeling of master station. A master station supervises the entire system. It collects information, such as voltage, current and frequency, generator output and on-off state of breakers in the controlled area. A sub-station can easily obtain information about other sub-stations by communicating with the master station. To accurately simulate dynamic behaviors of an SSCS, the master station model should be built according to network structure, generating scheme, load level, and so on. Such information can be used as input relays when we model the master station.

Model of Substation. A substation model collects such information as operation mode, network structure, calculates related interface power flow, identifies faults and achieves control functions through the master station model.

Fault Identification. Control measures of an SSCS include that generator unit tripping, load shedding, system splitting, etc. They are closely related to the fault type, therefore accurate identification of fault information is a key problem to modeling of control strategies. By using different switch signals and electrical quantities, we build various fault detection models according to actual demand from power systems. Fig.1 shows the model of instantaneous single-phase-to-ground fault below, and the corresponding reference numbers of virtual relays are given in brackets. 


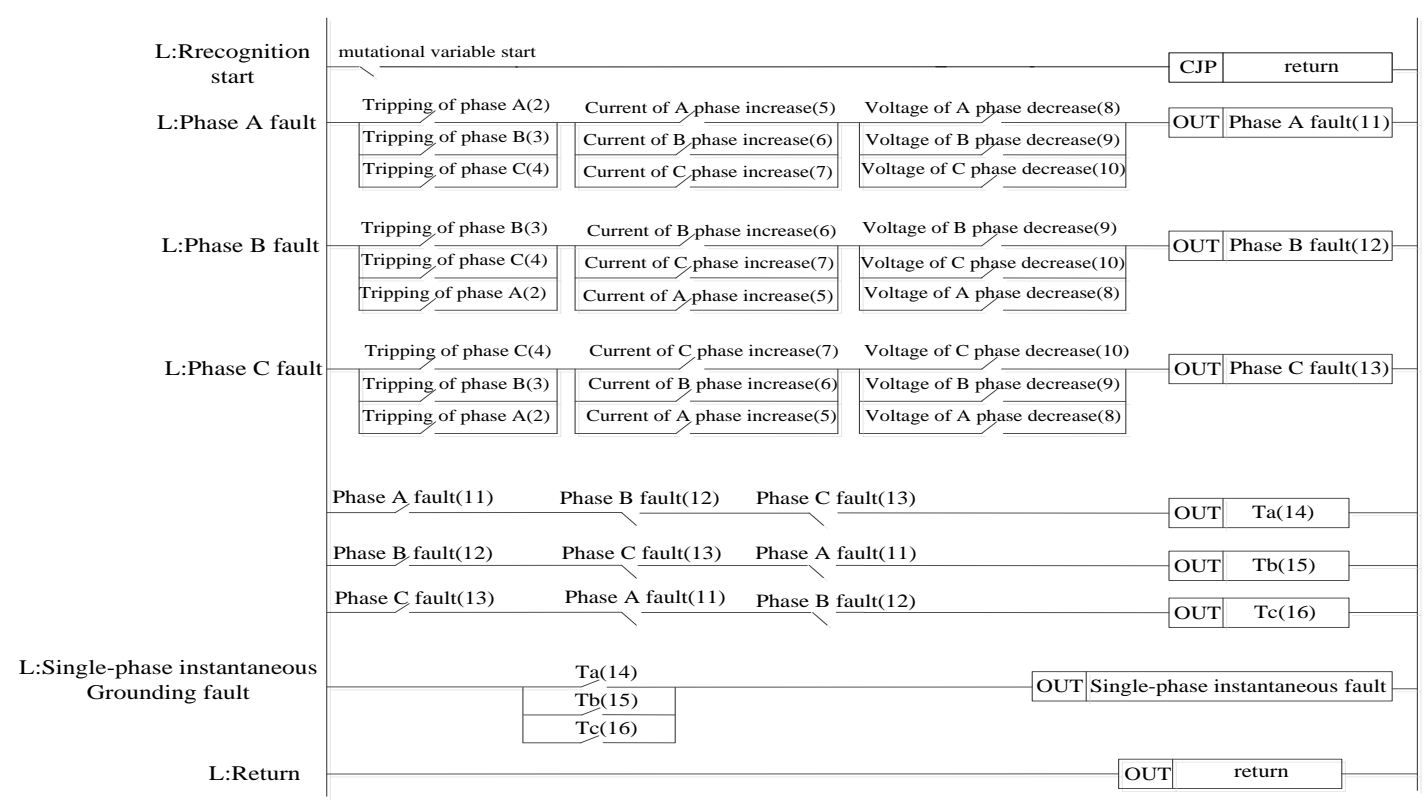

Fig.1 instantaneous single-phase-to-ground fault in transmission line

In the same way, we can set up models for single-phase-to-ground permanent fault, phase-to-phase fault, non-fault tripping of lines and transformers, circuit breaker failure, HVDC monopole block, HVDC bipolar bloc, and so on.

Control Strategies. Control strategies are the most important parts of an SSCS, which involve many functions such as operation mode detection, fault recognition and control measures. Accordingly, control strategy tables are normally of great complexity. Therefore, we develop an intuitive way to model a SSCS by using a strategy tree to save and check control strategies: root node->mode of connection ->mode of power flow ->mode of fault $->$ protection action ->control measures.

A strategy table includes control strategies for different operation mode. When a fault occurs, the simulation program will scan the strategy table of current operation mode. If trigger conditions are satisfied, the system will search and execute the corresponding control measures to ensure system stability.

Simulation Implementation. A simulation program is developed in this paper to model the performance of the master station and sub-stations. It includes dynamic component models, such as generators, excitation regulators and speed governors, together with models of buses and transmission lines. The SSCS model supervises its terminal devices at every integration step and gets real time data such as voltage, current, frequency and states of circuit breakers. When a terminal device starts, the SSCS will judge whether a fault happens and search corresponding control strategies. If conditions are satisfied, the SSCS will trig relevant control measures and change the topology of the network. And then, the dynamic simulation program will continue to iterate at next step to get the new state of the system. Thus the closed loop stability dynamic simulation can be realized.

\section{Simulation}

The schematic diagram of the case system is shown in fig.2. And the SSCS is modeled by using the method proposed in this paper. Control Strategies under different operation modes are checked. Due to the limited space, only the situation in sub-station 28 under a typical operation mode is discussed. 


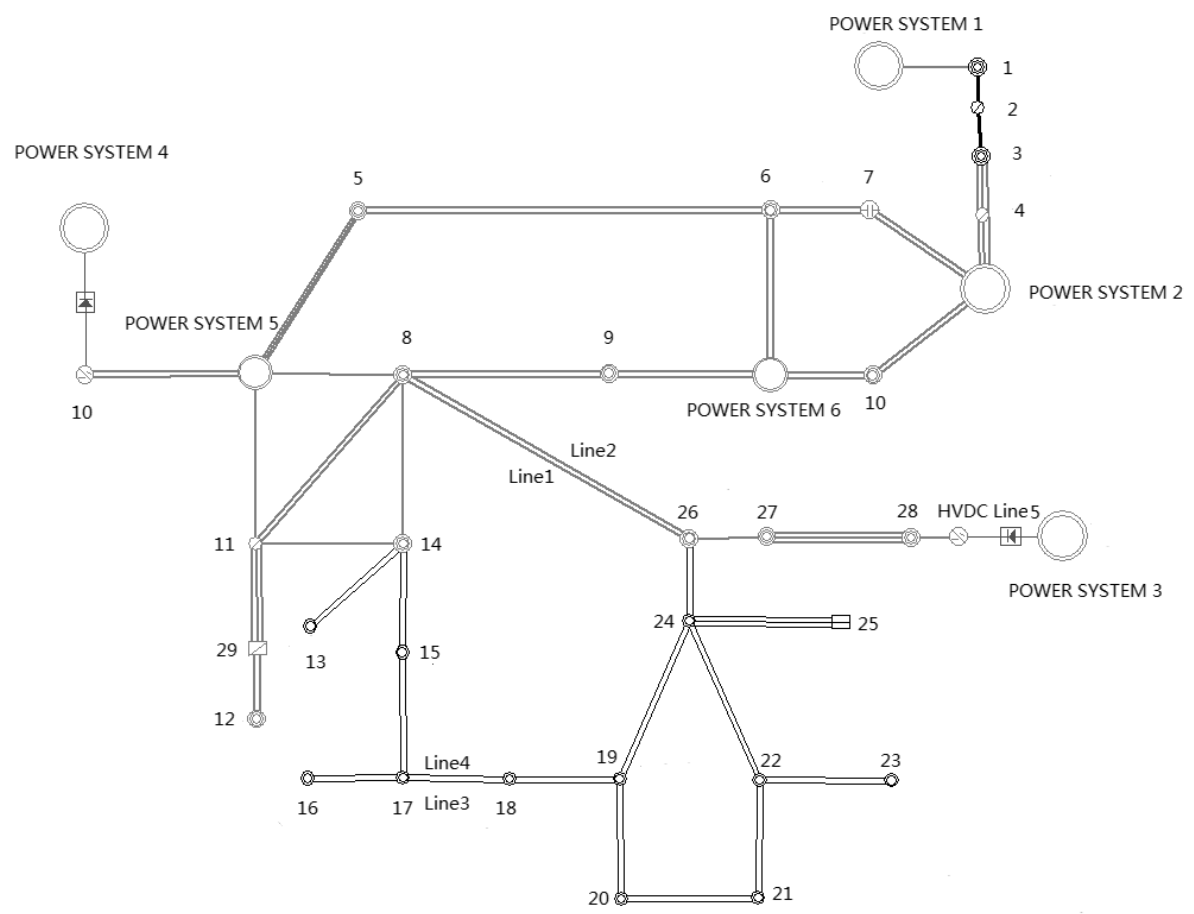

Fig.2 Schematic diagram of power system connection

When the simulation begins, the master station of the SSCS automatically identifies the operating mode according to the system parameters and network topology. A permanent fault occurs at $0.3 \mathrm{~s}$ on transmission Line 1 . At $0.4 \mathrm{~s}$, the relays at both ends of Line 1 act to clear the fault. However, Line2 is also cut off without reclose because of the malfunction of protections. The SSCS identifies the fault types, checks interface power flow, and then quickly searches and executes the appropriate control measures. As a result, the SSCS trips three generator units in plant 29 at $0.49 \mathrm{~s}$, cuts off the parallel transmission lines between buses 27 and 28 at $0.5 \mathrm{~s}$, and blocks HVDC Line 5 at $0.52 \mathrm{~s}$. Hence, the case system restores to stable gradually.

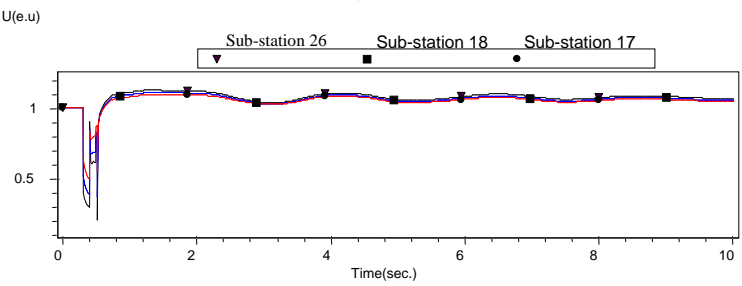

Fig.3 voltage near sub-station 26

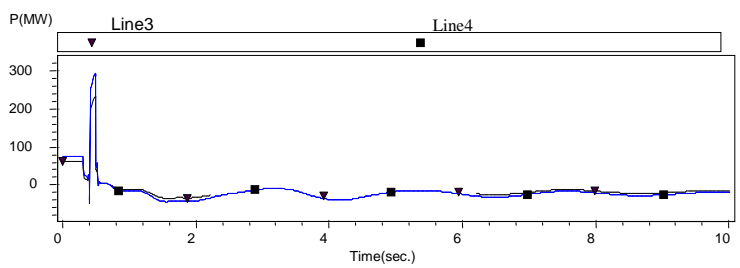

Fig.4 power of Line3 and Line4

Due to the correct operation of the SSCS, the voltage near sub-station 26 remained basically unchanged, and the $220 \mathrm{kV}$ transmission lines Line3 and Line4 are not overheated. It can be seen clearly in Fig.3 and Fig.4. The results show that the simulation program including SSCS models can represent the dynamic behavior of the real SSCS after a disturbance, and the control strategies can meet the requirements of the system stability. 
Although the performance of a SSCS with determined faults at definite time can also be realized by a great deal of calculation combined with repeated fault setting and data testing. It is difficult to achieve an accurate assessment of security and stability. Accurate models of SSCSs are very necessary in power system dynamic simulation, when it comes to relay actions caused by overload due to power flow transfer, or disconnection of power system components trigged by inverse-time characteristic relays and grid-related generator protections, especially in widespread power outages provoked by cascading failures.

\section{Summary}

A new modeling method of SSCS based on virtual relays is proposed in this paper. It can be used to build an SSCS model conforming to the actual power system in China. It can not only simulate the dynamic performance but also evaluate the safety of the power system more accurately. It helps to find out the reasons of serious accidents and research anti-accident measures to blackouts caused by cascading failures.

\section{References}

[1] Wu Wen-chuan, Sun Hong-bin . Zhang Bo-ming, et al. Simulation of automatic device based on user-defined model in integrated EMS / DTS. Automation of Electric Power Systems. 2000, 24(4) : 57-60.

[2] SUN Hong-bin, ZHANG Bo-ming, WU Wen-chuan, et al. A new dispatcher training simulator developed for Sub-transmission power network[J]. Automation of Electric Power Systems, 2001, 25(4): 49-52.

[3] WANG Wei-guo, DAI Wei, WAN Lei. Study of the simulation methods of protection relay and security automatic equipment in dispatcher training simulator (DTS). Automation of Electric Power Systems, 2003, 27(5) : 58-60.

[4] CUI Hong-bin, CHANG Xian-rong. Improvement of the simulation method about relay protection in DTS. Rrealy, 2006,34(1):22-26.

[5] DU Xin-wei, LIU Di-chen, YUAN Rong-xiang, et al. Virtual protection flexible modeling and simulation system. Automation of Electric Power Systems, 2008, 32(17): 55-60.

[6] WANG Le, WAN Qiu-lan. Extended Time Petri Net Applied in Action Logic Simulation of Protection and Automation Equipment in DTS. Automation of Electric Power Systems. 2005, 29(6) : 71-74.

[7] JIA Ning, CHANG Xian-rong. Development of the simulation module about relay protection in Dispatcher Training Simulator(DTS) . Realy, 2007, 35(23): 19-23.

[8] SUN Yuan-zhang, YANG Jun, ZHANG Xiao-dong, et al. Relay protection model for dynamic simulation of power system. Automation of Electric Power Systems. 2009, 33(20): 47-51.

[9] WU Guo-yang, SONG Xin-li, TANG Yong, et al. Modeling the Protective System for Dynamic Simulation of Power Systems. Automation of Electric Power Systems. 2010, 34(23):64-70. 